\title{
GENERALIZED FUNCTION TREATMENT OF THE ALFVÉN-GRAVITY WAVE PROBLEM
}

\author{
L. DEBNATH and K. VAJRAVELU \\ Department of Mathematics \\ East Carolina University \\ Greenville, N. C. 27834, U.S.A. \\ (Received September 30, 1982 and in revised form Apri1 12, 1983)
}

ABSTRACT. A study is made of the steady-state Alfvén-gravity waves in an inviscid incompressible electrically conducting fluid with an interface due to a harmonically oscillating pressure distribution acting on the interface. The generalized function method is employed to solve the problem in the fluid of infinite, finite and shallow depth. A unique solution of physical interest is derived by imposing the Sommerfeld radiation condition at infinity. Several limiting cases of physical interest are obtained from the present analysis. The physical significance of the solutions and their limiting cases are discussed. KEY (IORDS AND PHRASES. Alkvén-aravitu waves, generalized functions, Fourier transforms of distributions, water uaves, disnersion relations. 1980 MATHEMATICS SUBJECT CLASSIFICATION CONES. 76B15, 761105.

\section{INTRODUCTION.}

Steady-state surface wave problem in which the wave motion is simple harmonic in time is one of the most fundamental problems in the theory of surface wave phenomena in oceans. This is, indeed, a key problem for discussion of a variety of results and observations in water waves. However, it is, in general, not possible to derive a unique solution of the problem by imposing only bounded condition at infinity. In fact, sharper conditions such as the Sommerfeld radiation condition are required to obtain a unique solution of physical interest.

Several methods have been employed to solve the steady-state wave problem in order to obtain a unique solution of physical interest. These methods include (i) Lamb's fictitious damping force method $\Gamma 1^{7}$, (ii) Stoker's complicated complex variable method $\Gamma 27$, (iii) Lighthill's method $[37$ concerning an alternative way of applying the radiation condition and (iv) Debnath's generalized function method 147 . A11 these methods have been aimed at deriving a unique solution of physical interest, and in fact, led to the same solution.

An extensive usage of classical Fourier transform methods in water wave problems and other wave problems are well known and readily available in the existing 
literature (Stoker $\Gamma 27$, Sneddon $\Gamma 57$ ) on the subiects. However, Debnath $\lceil 47$ has clearly pointed out certain inherent difficulties involved in the steady-state water wave problems and in their method of solution by the classical Fourier analysis. These difficulties are mainly related to the existence, uniqueness, and the real singularies of the inverse Fourier integral solution of the problems. A careful study indicates that a new treatment of the steady-state surface wave problem by the generalized function method $\lceil 4\rceil$ can not only eliminate the inherent difficulties of the classical Fourier transform analysis, but also enable us to obtain unique solution of physical interest. Thus it seems to be an extremely useful device from the view point of sufficient generality. Another convincing point about this method is that there is neither any need for modification of the basic equations (Lamb $\Gamma 17$ ) nor any justification of the limit operation involved in the solution obtained by Lighthill's method $\Gamma 37$.

In spite of the tremendous progress on the steady-state surface wave problems in non-conducting fluids, hardly any attention has been given to the steady-state Alfvén-gravity wave problem in an electrically conducting fluid. The main purpose of this paper is to make a steady-state investigation of the Alfvén-gravity waves in an inviscid, incompressible electrically conducting liquid with an interface due to a harmonically oscillating pressure acting on the interface. A new generalized function method will be employed to solve this problem in the fluid of infinite, finite and shallow depth. A unique solution of physical interest is derived by imposing the Sommerfeld radiation condition at infinity. Several limiting cases of interest have been obtained from the present analysis. The physical significance of the solutions and their limiting cases are discussed.

2. MATHEMATICAL FORMULATION.

We consider the two-dimensional linearized steady-state Alfvén-gravity wave problem in an inviscid, incompressible electrically conducting fluid of uniform density $\rho$ with a free surface. In its undisturbed state, the field-free conducting fluid of constant depth $h$ occupies the region $-h \leq z \leq 0$ in the rectangular Cartisian coordinate system with the origin at the free surface, and the region $z>0$ is a vacuum under the action of constant magnetic field $\underline{B}_{0}=\left(B_{0}, 0,0\right)$.

We investigate the generation and propagation of magnetohydrodynamic surface waves in the fluid system due to a harmonically-oscillating pressure distribution acting at the interface $z=0$ in the form

$$
p(x, t)=\operatorname{Pf}(x) e^{i \omega t}
$$

where $P$ is a constant, $f(x)$ is a physically realistic arbitrary function of $x$, and $\omega$ is the fixed frequency.

In view of the fact that the motion is irrotational, there exists a velocity potential $\phi(x, z ; t)$ which satisfies the Laplace equation

$$
\nabla^{2} \phi \equiv \frac{\partial^{2} \phi}{\partial x^{2}}+\frac{\partial^{2} \phi}{\partial z^{2}}=0
$$

in $-\mathrm{h} \leq \mathrm{z} \leq 0$ and $-\infty<\mathrm{x}<\infty$. 
Outside the fluid the vacuum magnetic field must be a potential field so that the magnetic potential $\psi$ also satisfies the Laplace equation

$$
\nabla^{2} \psi \equiv \frac{\partial^{2} \psi}{\partial x^{2}}+\frac{\partial^{2} \psi}{\partial z^{2}}=0
$$

in $z>0$ and $-\infty<x<\infty$, with the boundary condition at infinity

$$
|\nabla \psi| \rightarrow 0 \text { as } z \rightarrow \infty
$$

The free surface dynamic and kinematic conditions in the linearized form are

$$
\left.\begin{array}{rl}
\phi_{t}+g n+\frac{B_{0}}{4 \pi \rho} \psi_{z} & =-\frac{1}{\rho} p(x, t) \\
n_{t} & =\phi_{z}
\end{array}\right\} \text { at } z=0,
$$

where $\eta \equiv \eta(x, t)$ is the vertical free surface elevation, and $g$ is the acceleration due to gravity.

The interfacial condition describes the constraint of frozen-in lines of force in the fluid on the vacuum magnetic field so that the interface remains a magnetic field line even in the disturbed state and is given by

$$
\frac{\partial \psi}{\partial z}=B_{0} \frac{\partial \eta}{\partial x} \quad \text { on } \quad z=0
$$

In the case of infinitely deep fluid, the bottom boundary condition is given by

$$
\phi \rightarrow 0 \quad \text { as } \quad z \rightarrow-\infty
$$

For the case of fluid of finite depth, the boundary condition at the rigid bottom is

$$
\phi_{z}=0 \text { on } \mathrm{z}=-\mathrm{h} \text {. }
$$

In addition, an appropriate radiation condition has to be imposed to ensure the uniqueness of the solution of the steady-state wave problem. This will be done later at appropriate places.

3. SOLUTION OF THE PROBLEM IN A FLUID OF INFINITE DEPTH.

As a trial, we assume that functions $\phi, \psi$ and $n$ possess ordinary Fourier transform with respect to $x$ defined by the integral

$$
\bar{\phi}(k, z ; t)=\frac{1}{\sqrt{2 \pi}} \int_{-\infty}^{\infty} e^{-i k x} \phi(x, z ; t) d x .
$$

Application of the Fourier transform method to equat 1 ons (2.1)-(2.7) gives solution for $\bar{\phi}, \bar{\psi}$ and $\bar{n}$ as

$$
\begin{aligned}
\bar{\phi}(k, z ; t) & =\frac{p i \omega \bar{f}(k) e^{i \omega t}+|k| z}{\rho D(\omega, k)}, \\
\bar{\psi}(k, z ; t) & =-\frac{P i|k| B_{o} \bar{f}(k) e^{i \omega t}-|k| z}{\rho D(\omega, k)},
\end{aligned}
$$




$$
\bar{n}(k, t)=\frac{p|k| \bar{f}(k) e^{i \omega t}}{\rho D(\omega, k)}
$$

where

$$
D(\omega, k) \equiv \omega^{2}-g|k|-a^{2} k^{2}
$$

and $a=B_{0} / \sqrt{4 \pi \rho}$ is the Alfvén wave velocity.

The use of the inverse Fourier transformation gives the integral solutions for $\phi, \psi$ and $n$ in the form

$$
\left[\begin{array}{l}
\phi \\
\psi \\
n
\end{array}\right]=\frac{1}{\sqrt{2 \pi}} \int_{-\infty}^{\infty} e^{i k x}\left[\begin{array}{l}
\bar{\phi} \\
\bar{\psi} \\
\bar{n}
\end{array}\right] d k
$$

It is noted that these inversion integrals are divergent because of the fact that the integrands of $(3.6 \mathrm{abc})$ have polar singularities at the zeros of $D(\omega, k)$ on the axis of integration. Consequently, the original assumption that functions $\phi, \psi$ and $n$ possess the Fourier transform in the ordinary sense was invalid. This is logical because a function which has a Fourier transform must tend to infinity, but we expect $\phi, \psi$ and $n$ to have wave-like behaviour, that is, bounded at infinity but not tending to zero at infinity.

To overcome this inherent difficulty, we treat the functions $\phi, \psi$ and $n$ as generalized functions in the sense of Lighthill $\$ 67$ in which case they do have the Fourier transform. Thus $(3.6 \mathrm{abc})$ represent valid inversion integrals in the generalized sense. So one can use the generalized function method to evaluate the integra1 ( $3.6 \mathrm{abc})$. It would be sufficient for determination of the principal features of the wave motions to evaluate the integral for $n(x, t)$. We next evaluate (3.6c) by using result (24) of Debnath and Resenblat 5.77 based on the generalized function method, that is, if $\overline{\mathrm{F}}(\mathrm{k})$ has a simple pole at $\mathrm{k}=\mathrm{k}_{1}$ in $\mathrm{a}<\mathrm{k}_{1}<\mathrm{b}$, then as $|x| \rightarrow \infty$,

$$
\left.\int_{a}^{b} \bar{F}(k) e^{i k x} d k \sim i \pi \operatorname{sgn} x e^{i k} 1^{x} \quad \text { (residue of } \bar{F}(k) \text { at } k=k_{1}\right)+0\left(\frac{1}{x} \mid\right) \text {. }
$$

Making use of the result, we evaluate integral (3.6c) written explicitly in the form

$$
\eta(x, t)=\frac{\rho e^{i \omega t}}{\rho \sqrt{2 \pi}} \int_{-\infty}^{\infty} \frac{|k| \bar{f}(k) e^{i k x} d k}{D(\omega, k)}
$$

to obtain the solution for $n(x, t)$ as $|x| \rightarrow \infty$ in the form

$$
\begin{aligned}
n(x, t) & \sim \frac{p \pi i e^{i \omega t}}{\rho \sqrt{2 \pi} a^{2}} \frac{\operatorname{sgn} x}{\left(k_{2}-k_{1}\right)}\left[k_{1} \bar{f}\left(k_{1}\right) e^{-i k_{1} x}-k_{2} \bar{f}\left(k_{2}\right) e^{-i k_{2} x}\right. \\
& \left.+k_{1} \bar{f}\left(k_{1}\right) e^{i k_{1} x}-k_{2} \bar{f}\left(k_{2}\right) e^{i k_{2} x}\right]+0\left(\left|\frac{1}{x}\right|\right)
\end{aligned}
$$


where $k_{1}$ and $k_{2}$ are the real roots of the disdersion equation

$$
D(\omega, k) \equiv \omega^{2}-g|k|-a^{2} k^{2}=0 \text {. }
$$

The solution (3.9) for the free surface elevation $n(x, t)$ contains four exponential terms -- two of them correspond to outgoing dispersive Alfvén-gravity waves from the source of disturbance and the other two represent incoming waves from infinity.

In order to achieve unique solution of physical interest, we impose the Sommerfeld radiation condition to eliminate the incoming wave terms present in the solution ( 3.9 ). This leads to the unique solution for $n(x, t)$ in the form

$$
n(x, t) \sim \frac{p \pi i e^{i \omega t}}{\rho a^{2} \sqrt{2 \pi}\left(k_{2}-k_{1}\right)}\left[k_{1} \bar{f}\left(k_{1}\right) e^{-i k_{1}|x|}-k_{2} \bar{f}\left(k_{2}\right) e^{i k_{2}|x|}\right]+0\left(\frac{1}{x} \mid\right) .
$$

This result corresponds to dispersive Alfvén-gravity waves characterized by the dispersion relation (3.10) which gives the phase velocity, $\mathrm{C}_{\mathrm{pm}}$ and the group velocity $c_{\text {gm }}$ as

$$
\begin{aligned}
& C_{p m}=\frac{\omega}{k}=\left(\frac{g}{k}+a^{2}\right)^{\frac{1}{2}}, \\
& C_{g m}=\frac{\partial \omega}{\partial k}=\frac{1}{2} \frac{g+2 a^{2} k}{\sqrt{g k+a^{2} k^{2}}} .
\end{aligned}
$$

In the absence of the external magnetic field $\underline{B}_{0}$, all these results reduce to those for pure gravity wave solution due to Debnath $4^{7}$. In particular, when $f(x) \equiv \delta(x)$, the Dirac delta distribution so that $\bar{f}(k)=\frac{1}{\sqrt{2 \pi}}$, we also recover Debnath's result for the steady-state gravity waves from (3.11) in the 1 imit a $\rightarrow 0$. 4. SOLUTION OF THE PROBLEM IN A FLUID OF FINITE DEPTH.

Application of the generalized Fourier transform to (2.1)-(2.6) combined with bottom boundary condition (2.8) gives solutions for $\bar{\phi}, \bar{\psi}$ and $\bar{\eta}$ as

$$
\begin{aligned}
& \bar{\phi}(k, z ; t)=\frac{P \operatorname{i\omega } \bar{f}(k) e^{i \omega t}}{\rho D_{\star}(\omega, k)} \frac{\operatorname{Cosh}|k|(z+h)}{\operatorname{Cosh}|k| h}, \\
& \bar{\psi}(k, z ; t)=-\frac{P i|k| B_{0} \bar{f}(k) \tanh |k| h \exp (i \omega t-|k| z)}{\rho D_{*}(\omega, k)}, \\
& \bar{n}(k, t)=\frac{P e^{i \omega t} \overline{f(k)}|k| \tanh |k| h}{\rho D_{*}(\omega, k)},
\end{aligned}
$$

where

$$
D_{*}(\omega, k)=\omega^{2}-\left(g|k|+a^{2} k^{2}\right) \tanh |k| h
$$

The inverse Fourier transformation enables us to obtain an explicit integra1 solution for $n(x, t)$ as 


$$
n(x, t)=\frac{P e^{i \omega t}}{2 \rho \omega \sqrt{2 \pi}} \int_{-\infty}^{\infty}|k| \tanh |k| h\left(\frac{1}{\alpha+\omega}-\frac{1}{\alpha-\omega}\right) \bar{f}(k) e^{i k x} d k,
$$

where $\alpha \equiv \alpha(k)$ is given by

$$
\alpha^{2}(k) \equiv\left(g|k|+a^{2} k^{2}\right) \tanh |k| h \quad .
$$

Making use of result (3.7), we can evaluate the wave integral (4.5). It turns out that the significant contribution to the solution for $n(x, t)$ as $|x| \rightarrow \infty$ comes from the poles of the integrand of (4.5). These poles are the roots of the equation $\alpha(k) \pm \omega=0$ and are readily found from the points of intersection of the curves $\zeta=\alpha(k)$ with the straight lines $\zeta= \pm \omega$. From the graphical representation of these curves, it turns out that real roots of the above equations are at $k= \pm k_{1}^{*}$, $\pm \mathrm{k}_{2}^{*}$.

Using the same formula (3.7), the wave integral (4.5) can easily be evaluated to obtain the solution as

$$
\begin{array}{r}
n(x, t) \sim \frac{P \pi i e^{i \omega t} \operatorname{sgn} x}{2 \rho \omega \sqrt{2 \pi}}\left[\bar{F}\left(k_{1}^{*}\right)\left\{e^{i k_{1} * x}+e^{-i k_{1} x_{x}}\right\}\right. \\
\left.-\bar{F}\left(k_{2} *\right)\left\{e^{i k_{2}^{*} x}+e^{-i k_{2} x_{x}}\right\}\right]+0\left(\left|\frac{1}{x}\right|\right),
\end{array}
$$

where

$$
\overline{\mathrm{F}}(\mathrm{k})=[\overline{\mathrm{f}}(\mathrm{k}) \mathrm{k} \tanh \mathrm{kh}] /\left(\frac{\mathrm{d} \alpha}{\mathrm{dk}}\right)
$$

As in the case of infinitely deep fluid, the solution (4.7) consists of both outgoing waves from the source of the disturbance and incoming waves from infinity. In order to eliminate the incoming solution, we require to impose the Sommerfeld radiation condition. This leads to the unique solution of physical interest in the form

$$
n(x, t)=\frac{P \pi i e^{i \omega t}}{2 \rho \omega \sqrt{2 \pi}}\left[\bar{F}\left(k_{1} *\right) e^{-i k_{1} x^{x}}-\bar{F}\left(k_{2} *\right) e^{i k_{2} * x}\right] .
$$

This solution also represents dispersive Alfvén-gravity waves characterized by the dispersion relation $\alpha^{2}(k)=\omega^{2}$ which gives the phase and the group velocities of the wave motions. In the limiting case of infinitely deep fluid $(k h+\infty)$, solution (4.9) reduces to result (3.11). On the other hand, when there is no external magnetic field $\left(B_{0} \rightarrow 0\right)$, these findings are in agreement with those obtained earlier by Debnath [4]. 
5. DISPERSIVE WAVES IN A VERY SHALLOW CONDUCTING FLUID.

In this case $(k h \rightarrow 0) \tanh |k| h \sim|k| h$ so that $\alpha^{2}(k) \sim c^{2} k^{2}+a^{2} h|k| k^{2}$; $c^{2}=g h$. Consequently, the integral solution (4.5) can easily be simplified to obtain an explicit representation for $n(x, t)$

$$
\eta(x, t)=-\frac{P e^{i \omega t}}{\rho a^{2} \sqrt{2 \pi}} \int_{0}^{\infty} \frac{k^{2} \bar{f}(k)}{k^{3}+B k^{2}+D}\left(e^{i k x}+e^{-i k x}\right) d k,
$$

where

$$
B=\frac{c^{2}}{h a^{2}}>0 \text { and } \quad D=-\frac{\omega^{2}}{h a^{2}}<0 \text {. }
$$

The significant contribution to the solution for $n(x, t)$ comes from real polar singularities of the integrand in (5.1). The singularities of the integrand in (5.1) are the roots of the cubic equation for $k$ in the form

$$
\mathrm{k}^{3}+\mathrm{Bk}^{2}+\mathrm{D}=0 \text {. }
$$

Since the constant form in (5.3) is negative, this cubic equation must, therefore, allow at least one positive real root, $k=k_{0}$. This lead to factorize (5.3) in the form

$$
\left(k-k_{0}\right)\left(k^{2}+2 \alpha k+\beta\right)=0 .
$$

By comparison with equation (5.3), it turns out that

$$
\alpha=-\frac{\mathrm{D}}{2 \mathrm{k}_{0}{ }^{2}}>0 \quad \text { and } \quad \beta=-\frac{\mathrm{D}}{\mathrm{k}_{0}}>0
$$

It can then readily be shown that the two remaining roots of (5.4) are either real and negative or complex conjugate with negative real parts.

A simple inspection reveals that the significant contribution to the solution for $n(x, t)$ comes from the positive real poles of the cubic (5.4). Following the same procedure used earlier, the integral (5.1) can easily be evaluated to obtain the solution in the form

$$
\eta(x, t) \sim-\frac{p e^{i \omega t}(\pi i \operatorname{sgn} x)}{\rho a^{2} \sqrt{2 \pi}} \frac{k_{0}^{2} \bar{f}\left(k_{0}\right)}{\left(k_{0}^{2}+2 \alpha k_{0}+\beta\right)}\left(e^{i k_{0} x}+e^{-i k_{0} x}\right) .
$$

By imposing the radiation condition, a unique solution of physical interest has the form

$$
n(x, t) \sim-\frac{P \pi i \operatorname{sgn} x}{\rho a^{2} \sqrt{2 \pi}} \frac{k_{0}^{2} \bar{f}\left(k_{0}\right)}{\left(k_{0}^{2}+2 \alpha k_{0}+\beta\right)} e^{i\left(\omega t-k_{0}|x|\right)} .
$$

This solution represents the dispersive Alfvén-gravity waves characterized by the dispersion equation (5.3). Hence, the phase and the group velocities of the waves can readily be calculated. In the absence of the magnetic field $\left(\mathrm{B}_{0} \rightarrow 0\right.$ and $\left.\mathrm{a} \rightarrow 0\right)$, the above result (5.7) reduces to the corresponding solution in a shallow non-conducting liquid, and shows a striking contrast to the non-dispersive shallow water waves. 
6. CLOSING REMARRS.

The above entire analysis has been carried out for an arbitrary function $f(x)$ involved in the applied pressure given by (2.1). Some simple functional form for $f(x)$ can easily be chosen to illustrate the general theory presented in this paper. However, the present study has revealed the principal features of the dispersive Alfvén-gravity wave motions in a conducting liquid.

ACKNOWLEDGEMENT. This work was partially supported by a research grant from East Carolina University.

\section{REFERENCES}

1. LAMB, H. Hydrodynamics, Cambridge University Press, London, 1932.

2. STOKER, J. J. Water Waves, Interscience Publishers, New York, 1957.

3. LIGHTHILL, M. J. Studies on Magnetohydrodynamic Waves and Other Anisotropic Wave Motions, Phil. Trans. Roy. Soc. A252 (1960) 397-430.

4. DEBNATH, L. A New Treatment of the Steady-State Wave Problem - I, Proc. Nat. Institute Sci., India A35 (1969) 502-506.

5. SNEDDON, I.N. Fourier Transforms, McGraw Hill Publishing Company, 1952.

6. LIGHTHILL, M. J. An Introduction to Fourier Analysis and Generalized Functions, Cambridge University Press, 1958.

7. DEBNATH, L., and ROSENBLAT, S. The U1timate Approach to the Steady-State in the Generation of Waves on a Running Stream, Quart. Jour. Mech and App1. Math. 22 (1969) 221-231. 


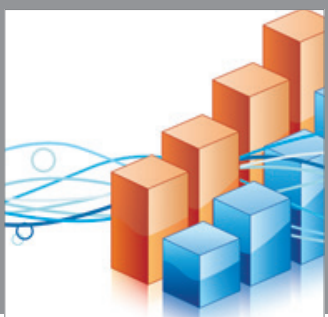

Advances in

Operations Research

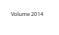

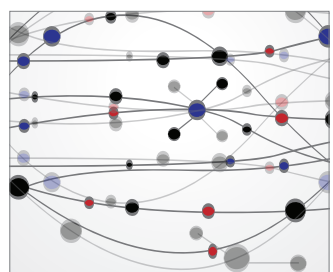

\section{The Scientific} World Journal
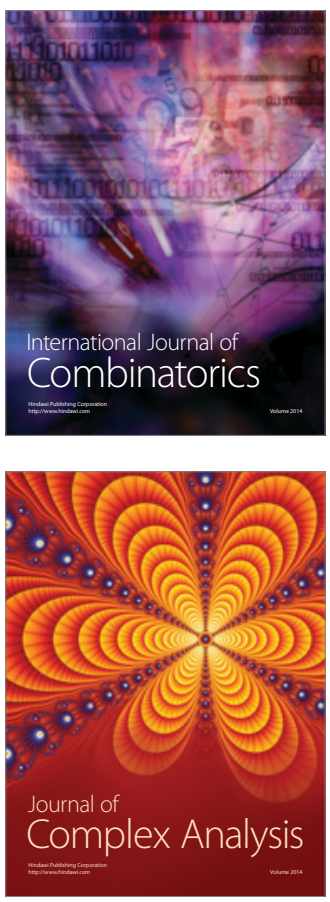

International Journal of

Mathematics and

Mathematical

Sciences
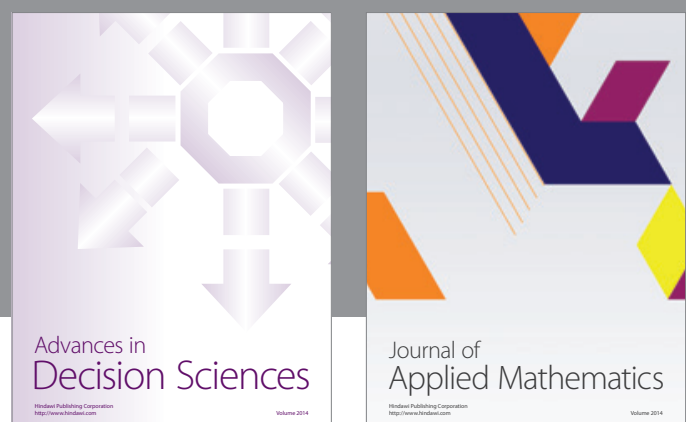

Journal of

Applied Mathematics
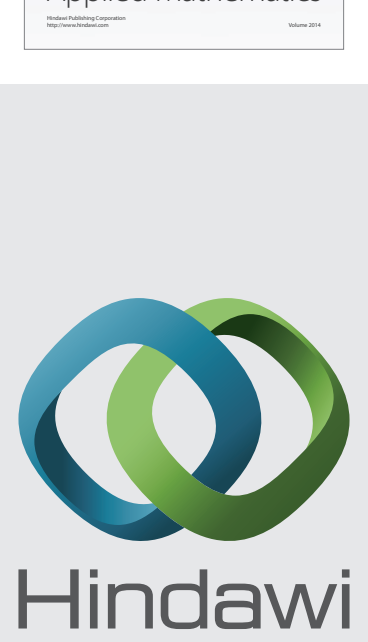

Submit your manuscripts at http://www.hindawi.com
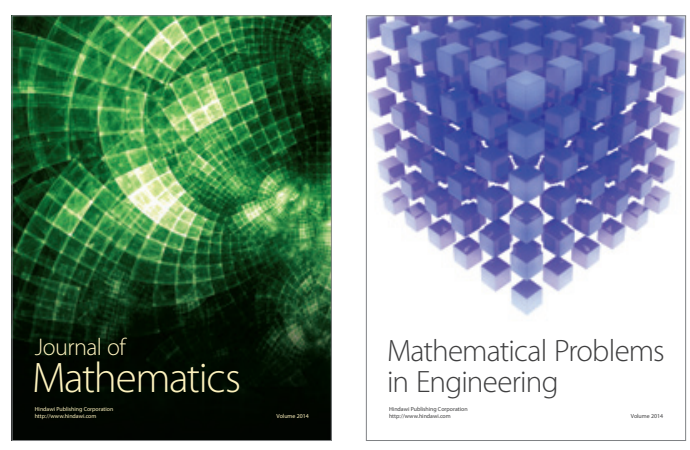

Mathematical Problems in Engineering
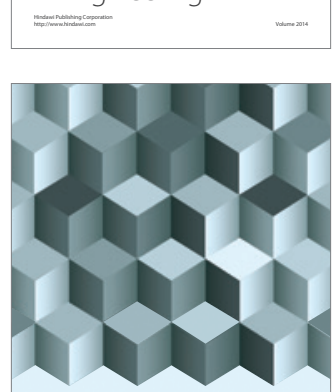

Journal of

Function Spaces
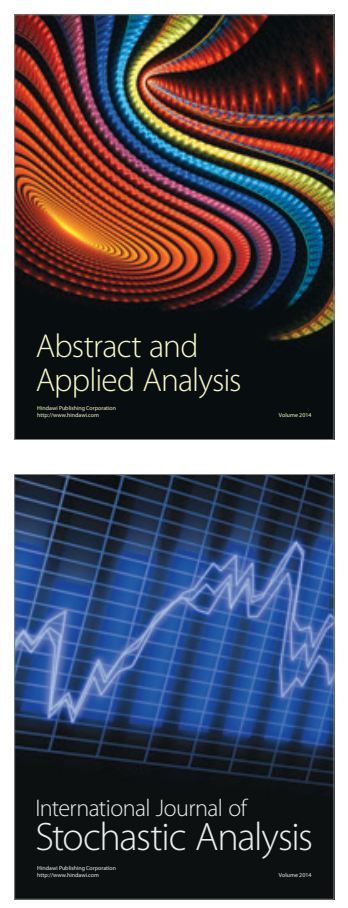

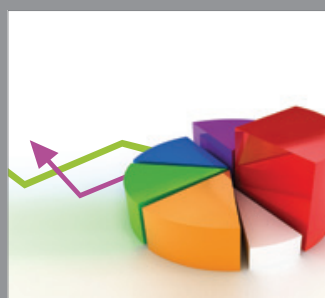

ournal of

Probability and Statistics

Promensencen
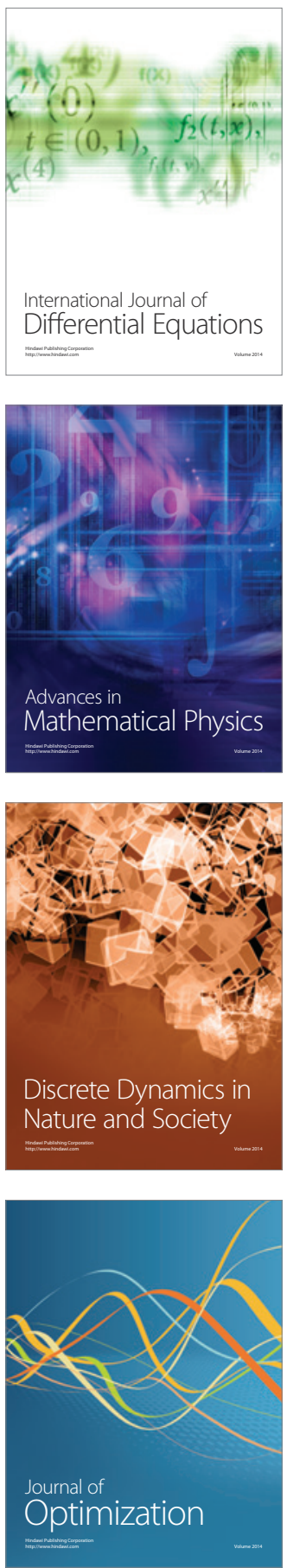